\title{
Gauss-Bonnet black holes supporting massive scalar field configurations: the large-mass regime
}

\author{
Shahar Hod ${ }^{1,2, a}$ \\ ${ }^{1}$ The Ruppin Academic Center, 40250 Emeq Hefer, Israel \\ 2 The Hadassah Academic College, 91010 Jerusalem, Israel
}

Received: 4 November 2019 / Accepted: 18 November 2019 / Published online: 25 November 2019

(C) The Author(s) 2019

\begin{abstract}
It has recently been demonstrated that black holes with spatially regular horizons can support external scalar fields (scalar hairy configurations) which are non-minimally coupled to the Gauss-Bonnet invariant of the curved spacetime. The composed black-hole-scalar-field system is characterized by a critical existence line $\alpha=\alpha\left(\mu r_{\mathrm{H}}\right)$ which, for a given mass of the supported scalar field, marks the threshold for the onset of the spontaneous scalarization phenomenon [here $\left\{\alpha, \mu, r_{\mathrm{H}}\right\}$ are respectively the dimensionless non-minimal coupling parameter of the field theory, the proper mass of the scalar field, and the horizon radius of the central supporting black hole]. In the present paper we use analytical techniques in order to explore the physical and mathematical properties of the marginally-stable composed black-hole-linearized-scalar-field configurations in the eikonal regime $\mu r_{\mathrm{H}} \gg 1$ of large field masses. In particular, we derive a remarkably compact analytical formula for the critical existence-line $\alpha=\alpha\left(\mu r_{\mathrm{H}}\right)$ of the system which separates bare Schwarzschild black-hole spacetimes from composed hairy (scalarized) black-hole-field configurations.
\end{abstract}

\section{Introduction}

The mathematically elegant no-hair theorems presented in [1-4] have revealed the physically important fact that, within the framework of classical general relativity, spherically symmetric black holes with regular horizons cannot support external static matter configurations which are made of scalar fields with minimal coupling to gravity. As explicitly proved in [5-7], the intriguing no-hair property of static black holes can also be extended to the physical regime of scalar matter fields which are characterized by a non-trivial (non-minimal) coupling to the Ricci curvature scalar of the corresponding spherically symmetric spacetimes.

\footnotetext{
a e-mail: shaharhod@gmail.com
}

Interestingly, later developments [8-14] have revealed the intriguing fact that spatially regular hairy matter configurations which are made of scalar fields with non-minimal couplings to the Gauss-Bonnet curvature invariant $\mathcal{G}$ may be supported in curved black-hole spacetimes. In particular, it has been proved [12-14] that, in extended Scalar-TensorGauss-Bonnet theories whose actions contain a non-trivial field-curvature coupling term of the form $f(\phi) \mathcal{G}$ [15], black holes with regular horizons may support scalar fields with non-trivial spatial profiles (see [16-18] for the physically related model of spontaneously scalarized charged blackhole spacetimes which owe their existence to a non-trivial coupling between the external scalar field and the electromagnetic field tensor of the central supporting charged black hole).

In a physically realistic field theory, the spontaneous scalarization phenomenon should be characterized by a nontrivial coupling function $f(\phi)$ whose mathematical form allows the existence of bare (non-scalarized) black-hole solutions in the weak-coupling regime [12-14]. Specifically, the physically important studies presented in [12-14] have considered Scalar-Tensor-Gauss-Bonnet theories whose coupling functions are characterized by the limiting behavior $f(\phi \rightarrow 0) \propto \alpha \phi^{2}$ in the weak-field regime. Here the physical parameter $\alpha$ is the dimensionless coupling constant of the non-trivial field theory [see Eq. (10) below].

Intriguingly, it has recently been proved [19] that, for non-minimally coupled massive scalar fields, the composed black-hole-field system is characterized by a critical existence-line $\alpha=\alpha\left(\mu r_{\mathrm{H}}\right)$ which separates bare Schwarzschild black holes from hairy (scalarized) blackhole-field solutions of the field equations (here $\mu$ is the proper mass of the supported scalar field and $r_{\mathrm{H}}$ is the horizon radius of the central black hole). In particular, the existence-line of the system corresponds to linearized marginally-stable scalar field configurations which are supported by central Schwarzschild black holes. [In the physics literature $[9,20-$ 
22], the supported linearized scalar field configurations are usually called scalar 'clouds' in order to distinguish them from self-gravitating (non-linear) hairy matter configurations]. Interestingly, the numerical results presented in [19] have revealed the fact that, for a given value of the dimensionless coupling parameter $\alpha$, the horizon radius (mass) of the central supporting black hole is a monotonically decreasing function of the mass of the supported scalar field.

The main goal of the present paper is to explore, using analytical techniques, the physical and mathematical properties of the composed Schwarzschild-black-hole-nonminimallycoupled-linearized-massive-scalar-field cloudy configurations In particular, using a WKB analysis in the dimensionless large-mass $\mu r_{\mathrm{H}} \gg 1$ regime, we shall derive a resonance formula that provides a remarkably compact analytical description of the critical existence-line $\alpha=\alpha\left(\mu r_{\mathrm{H}}\right)$ of the composed Schwarzschild-black-hole-massive-scalarfield system. Interestingly, the derived resonance formula [see Eq. (24) below] would provide a simple analytical explanation for the numerically observed [19] monotonic behavior of the function $r_{\mathrm{H}}=r_{\mathrm{H}}(\mu ; \alpha)$ along the critical existenceline of the system.

\section{Description of the system}

We shall study analytically the discrete resonant spectrum which characterizes the composed Schwarzschildblack-hole-linearized-massive-scalar-field configurations in the physical regime of large field masses. As shown numerically in [12-14, 19], the spatially regular cloudy field configurations owe their existence to their non-trivial coupling to the Gauss-Bonnet invariant $\mathcal{G} \equiv R_{\mu \nu \rho \sigma} R^{\mu \nu \rho \sigma}-4 R_{\mu \nu} R^{\mu \nu}+R^{2}$ of the curved spacetime. The black-hole spacetime is characterized by the spherically-symmetric curved line element [23]

$$
d s^{2}=-h(r) d t^{2}+\frac{1}{h(r)} d r^{2}+r^{2}\left(d \theta^{2}+\sin ^{2} \theta d \phi^{2}\right),
$$

where

$h(r)=1-\frac{r_{\mathrm{H}}}{r}$.

Here $r_{\mathrm{H}}=2 M$ is the horizon radius of the central supporting Schwarzschild black hole of mass $M$.

The composed black-hole-field system is characterized by the action $[12,13,19,24]$

$S=\frac{1}{2} \int d^{4} x \sqrt{-g}\left[R-\frac{1}{2} \nabla_{\alpha} \phi \nabla^{\alpha} \phi-\frac{1}{2} \mu^{2} \phi^{2}+f(\phi) \mathcal{G}\right]$, where the radius-dependent Gauss-Bonnet curvature invariant of the Schwarzschild black-hole spacetime is given by

$\mathcal{G}=\frac{12 r_{\mathrm{H}}^{2}}{r^{6}}$

The scalar function $f(\phi)$ in (3) controls the non-minimal coupling between the Gauss-Bonnet invariant of the curved spacetime and the massive scalar field. As shown in [12, $13,19]$, in order to guarantee the existence of bald (nonscalarized) black-hole solutions in the field theory, this coupling function should have the universal leading-order quadratic behavior

$f(\phi)=\frac{1}{8} \eta \phi^{2}$

in the linearized regime. The physical parameter $\eta$, which controls the strength of the non-trivial quadratic coupling between the massive scalar field and the Gauss-Bonnet curvature invariant, has the dimensions of length ${ }^{2}$.

Using the functional expression [25]

$\phi(r, \theta, \phi)=\sum_{l m} \frac{\psi_{l m}(r)}{r} Y_{l m}(\theta) e^{i m \phi}$

for the non-minimally coupled static scalar field and defining the tortoise radial coordinate $y$ by the relation [26]

$\frac{d r}{d y}=h(r)$,

one finds that the spatial behavior of the supported massive scalar field configurations in the Schwarzschild black-hole spacetime (1) is determined by the Schrödinger-like ordinary differential equations. [12,13,19]

$\frac{d^{2} \psi}{d y^{2}}-V \psi=0$

where $[12,13,19]$

$V(r)=\left(1-\frac{r_{\mathrm{H}}}{r}\right)\left[\frac{l(l+1)}{r^{2}}+\frac{r_{\mathrm{H}}}{r^{3}}+\mu^{2}-\frac{\alpha r_{\mathrm{H}}^{4}}{r^{6}}\right]$.

Here

$\alpha \equiv 3 \eta r_{\mathrm{H}}^{2}$

is the dimensionless non-trivial coupling parameter of the composed black-hole-massive-scalar-field system.

The Schrödinger-like Eq. (8) with its effective radial potential (9), supplemented by the physically motivated 
boundary conditions of exponentially decaying scalar eigenfunctions at spatial infinity and a spatially regular functional behavior at the black-hole horizon [12,13,19],

$\psi(r \rightarrow \infty) \sim r^{-1} e^{-\mu r} \rightarrow 0 ; \quad \psi\left(r=r_{\mathrm{H}}\right)<\infty$,

determine the discrete resonant spectrum $\left\{\alpha_{n}\left(\mu, r_{\mathrm{H}}\right)\right\}_{n=0}^{n=\infty}$ which characterizes the composed cloudy black-holenonminimally-coupled-linearized-massive-scalar-field configurations. In particular, the fundamental resonant mode, $\alpha_{0}=\alpha_{0}\left(\mu, r_{\mathrm{H}}\right)$, determines the critical existence-line of the field theory in the curved black-hole spacetime.

\section{The discrete resonant spectrum of the composed black-hole-linearized-massive-scalar-field system: A WKB analysis}

In the present section we shall use analytical techniques in order to study the discrete resonant spectrum $\left\{\alpha_{n}\left(\mu, r_{\mathrm{H}}\right)\right\}_{n=0}^{n=\infty}$ of the dimensionless scalar-Gauss-Bonnet coupling parameter which characterizes the composed black-hole-linearizedmassive-scalar-field configurations in the large-mass regime

$\mu r_{\mathrm{H}} \gg \max \{1, l\}$.

We first point out that, in terms of the tortoise coordinate $y$ [see Eq. (7)], the Schrödinger-like radial differential Eq. (8) has a mathematical form which is amenable to a standard WKB analysis. In particular, a standard second-order WKB analysis for the spatially regular bound-state resonances of the Schrödinger-like ordinary differential equation (7) yields the well-known quantization condition [27-30]

$$
\int_{y_{-}}^{y_{+}} d y \sqrt{-V(y ; \alpha)}=\left(n-\frac{1}{4}\right) \cdot \pi ; \quad n=1,2,3, \ldots
$$

The two boundaries $\left\{y_{-}, y_{+}\right\}$of the WKB integral relation (13) are determined by the classical turning points of the radial binding potential (9) [that is, $V\left(y_{-}\right)=V\left(y_{+}\right)=0$ ]. The integer $n$ is the resonance parameter which characterizes the discrete bound-state resonant modes of the composed black-hole-nonminimally-coupled-massive-scalar-field system.

Taking cognizance of the differential relation (7), one can express the WKB integral relation (13), which characterizes the composed black-hole-linearized-massive-scalarfield configurations, in the form

$$
\int_{r_{-}}^{r_{+}} d r \frac{\sqrt{-V(r ; \alpha)}}{h(r)}=\left(n-\frac{1}{4}\right) \cdot \pi ; \quad n=1,2,3, \ldots
$$

The radial turning points $\left\{r_{-}, r_{+}\right\}$of the binding potential (9) are determined by the two polynomial relations

$$
1-\frac{r_{\mathrm{H}}}{r_{-}}=0
$$

and

$\frac{l(l+1)}{r_{+}^{2}}+\frac{r_{\mathrm{H}}}{r_{+}^{3}}+\mu^{2}-\frac{\alpha r_{\mathrm{H}}^{4}}{r_{+}^{6}}=0$.

As we shall now show explicitly, the WKB integral relation (14) can be studied analytically in the large-mass regime (12). In particular, defining the dimensionless radial coordinate

$x \equiv \frac{r-r_{\mathrm{H}}}{r_{\mathrm{H}}}$,

one can expand the effective binding potential of the composed black-hole-massive-field system in the form

$V[x(r)]=-\left(\frac{\alpha}{r_{\mathrm{H}}^{2}}-\mu^{2}\right) \cdot x+\left(\frac{7 \alpha}{r_{\mathrm{H}}^{2}}-\mu^{2}\right) \cdot x^{2}+O\left(x^{3}\right)$.

The near-horizon radial potential (18) has the form of an effective binding potential. In particular, from (18) one finds that the two turning points $\left\{x_{-}, x_{+}\right\}$of the WKB integral relation (14) are given by the simple dimensionless functional expressions

$x_{-}=0$

and

$x_{+}=\frac{\frac{\alpha}{r_{\mathrm{H}}^{2}}-\mu^{2}}{\frac{7 \alpha}{r_{\mathrm{H}}^{2}}-\mu^{2}}$.

Taking cognizance of Eqs. (17), (18), (19), and (20), one finds that, in the large-mass regime (12), the WKB integral equation (14) can be approximated by [31]

$\sqrt{\alpha-\mu^{2} r_{\mathrm{H}}^{2}} \int_{0}^{x_{+}} d x \sqrt{\frac{1}{x}-\frac{1}{x_{+}}}=\left(n-\frac{1}{4}\right) \cdot \pi ; n=1,2,3, \ldots$.

Interestingly, and most importantly for our analysis, the integral on the 1.h.s of Eq. (21) can be evaluated analytically to yield the WKB resonance relation

$$
\frac{\alpha-\mu^{2} r_{\mathrm{H}}^{2}}{2 \sqrt{7 \alpha-\mu^{2} r_{\mathrm{H}}^{2}}}=n-\frac{1}{4} ; \quad n=1,2,3, \ldots .
$$


The solution of the polynomial equation (22) for the dimensionless coupling parameter $\alpha$ of the composed blackhole-massive-field theory is given by the rather cumbersome expression

$$
\begin{aligned}
\alpha_{n}= & \mu^{2} r_{\mathrm{H}}^{2}+14\left(n+\frac{3}{4}\right)^{2} \\
& +2 \sqrt{6 \mu^{2} r_{\mathrm{H}}^{2}\left(n+\frac{3}{4}\right)^{2}+49\left(n+\frac{3}{4}\right)^{4}} ; \\
& n=0,1,2, \ldots .
\end{aligned}
$$

In the large-mass $\mu r_{\mathrm{H}} \gg n+1$ regime, the resonance spectrum (23) can be approximated by the compact analytical relation

$\alpha_{n}=\mu^{2} r_{\mathrm{H}}^{2} \cdot\left[1+\frac{2 \sqrt{6}\left(n+\frac{3}{4}\right)}{\mu r_{\mathrm{H}}}\right] ; \quad n=0,1,2, \ldots$

The discrete resonance spectrum (24) of the non-minimal coupling parameter $\alpha$ characterizes the cloudy Schwarzschildblack-hole-massive-scalar-field configurations in the eikonal large-mass regime (12).

\section{Summary}

The recently published highly interesting works $[12-14,19]$ have explicitly proved that, in some field theories, black holes may support external matter configurations (hair) made of scalar fields, a phenomenon which is known by the name black-hole spontaneous scalarization. In particular, it has been demonstrated numerically $[12-14,19]$ that spatially regular (massless as well as massive) scalar fields with nontrivial couplings to the Gauss-Bonnet curvature invariant may be supported by central black holes with regular horizons.

Intriguingly, the numerical results presented in [12-14, 19] have revealed the fact that the dimensionless physical parameter $\alpha$, which controls the non-trivial coupling between the Gauss-Bonnet invariant of the curved spacetime and the supported scalar matter configurations, is characterized by a discrete resonant spectrum $\left\{\alpha_{n}\right\}_{n=0}^{n=\infty}$ which corresponds to black holes that support spatially regular nonminimally coupled linearized scalar field configurations.

In the present paper we have used analytical techniques in order to explore the physical properties of the spontaneously scalarized hairy black-hole spacetimes in the regime of cloudy (linearized) supported field configurations. In particular, we have derived the compact WKB analytical formula (24) for the discrete resonant spectrum which characterizes the non-trivial coupling parameter $\alpha$ of the composed black-hole-massive-scalar-field theory in the physical regime $\mu r_{\mathrm{H}} \gg 1$ of large field masses.
Finally, it is worth pointing out that one may obtain from the analytically derived resonance spectrum (24) the remarkably compact formula [32]

$\mu r_{\mathrm{H}_{\max }}(\alpha)=\sqrt{\alpha+\frac{27}{8}}-\sqrt{\frac{27}{8}}$ for $\alpha \gg 1$

for the critical existence-line which characterizes the hairy Schwarzschild-black-hole-massive-scalar-field configurations. The $\alpha$-dependent critical line (25) for the masses of the supported non-minimally coupled scalar fields marks, in the large-mass $\mu r_{\mathrm{H}} \gg 1$ regime, the boundary between bald Schwarzschild black-hole spacetimes and spontaneously scalarized hairy black-hole-scalar-field spacetimes. In particular, for a non-trivial field theory with a given value of the physical coupling parameter $\alpha$ and for a given mass (radius) of the central supporting black hole, the hairy blackhole-nonminimally-coupled-massive-scalar-field configurations are characterized by the mass inequality $\mu(\alpha) \leq$ $\mu_{\max }(\alpha)$.

Interestingly, the analytically derived formula (25) for the critical existence-line of the system implies, in agreement with the important numerical results presented in [19], that, for a given value of the dimensionless coupling parameter $\alpha$, the mass $M$ (horizon radius $r_{\mathrm{H}}$ ) of the central supporting black hole is a monotonically decreasing function of the mass $\mu$ of the nonminimally coupled scalar field.

Acknowledgements This research is supported by the Carmel Science Foundation. I would like to thank Yael Oren, Arbel M. Ongo, Ayelet B. Lata, and Alona B. Tea for helpful discussions.

Data Availability Statement This manuscript has no associated data or the data will not be deposited. [Authors' comment: I would like to emphasize that all relevant physical and mathematical calculations are explicitly presented in this paper.]

Open Access This article is distributed under the terms of the Creative Commons Attribution 4.0 International License (http://creativecomm ons.org/licenses/by/4.0/), which permits unrestricted use, distribution, and reproduction in any medium, provided you give appropriate credit to the original author(s) and the source, provide a link to the Creative Commons license, and indicate if changes were made.

Funded by SCOAP ${ }^{3}$.

\section{References}

1. J.D. Bekenstein, Phys. Rev. D 5, 1239 (1972)

2. C.A.R. Herdeiro, E. Radu, Int. J. Mod. Phys. D 24, 1542014 (2015)

3. T.P. Sotiriou, Class. Quantum Gravity 32, 214002 (2015)

4. T.P. Sotiriou, V. Faraoni, Phys. Rev. Lett. 108, 081103 (2012)

5. A.E. Mayo, J.D. Bekenstein, Phys. Rev. D 54, 5059 (1996)

6. S. Hod, Phys. Lett. B 771, 521 (2017)

7. S. Hod, Phys. Rev. D 96, 124037 (2017)

8. E. Babichev, C. Charmousis, JHEP 08, 106 (2014)

9. C.A.R. Herdeiro, E. Radu, Phys. Rev. Lett. 112, 221101 (2014)

10. T.P. Sotiriou, S.-Y. Zhou, Phys. Rev. Lett. 112, 251102 (2014) 
11. T.P. Sotiriou, S.-Y. Zhou, Phys. Rev. D 90, 124063 (2014)

12. D.D. Doneva, S.S. Yazadjiev, Phys. Rev. Lett. 120, 131103 (2018)

13. H.O. Silva, J. Sakstein, L. Gualtieri, T.P. Sotiriou, E. Berti, Phys. Rev. Lett. 120, 131104 (2018)

14. P.V.P. Cunha, C.A.R. Herdeiro, E. Radu, Phys. Rev. Lett. 123, 011101 (2019)

15. Here $\phi$ is the non-minimally coupled scalar field

16. C.A.R. Herdeiro, E. Radu, N. Sanchis-Gual, J.A. Font, Phys. Rev. Lett. 121, 101102 (2018)

17. P.G.S. Fernandes, C.A.R. Herdeiro, A.M. Pombo, E. Radu, N. Sanchis-Gual, Class. Quantum Gravity 36, 134002 (2019)

18. S. Hod, Phys. Lett. B 798, 135025 (2019)

19. C.F.B. Macedo, J. Sakstein, E. Berti, L. Gualtieri, H.O. Silva, T.P. Sotiriou, Phys. Rev. D 99, 104041 (2019)

20. S. Hod, Phys. Rev. D 86, 104026 (2012). arXiv:1211.3202

21. S. Hod, Eur. Phys. J. C 73, 2378 (2013). arXiv:1311.5298

22. S. Hod, Phys. Rev. D 90, 024051 (2014). arXiv:1406.1179

23. We shall use natural units in which $8 \pi G=c=1$

24. One may also include a quartic self-interaction term of the form $-\lambda \phi^{4} / 2$ in the action [19]. However, as explicitly shown in [19], the black-hole-linearized-scalar-field configurations, which mark the threshold for the black-hole spontaneous scalarization phenomenon and are the main focus of the present paper, are independent of the value of the higher-order parameter $\lambda$

25. The parameters $\{l, m\}$ (with $l \geq 0$ and $-l \leq m \leq l$ ) in the scalar field decomposition (6) are respectively the spherical and azimuthal harmonic indices. For brevity, we shall henceforth omit these integer indices
26. Note that the differential relation (7) maps the semi-infinite radial regime $r \in\left[r_{\mathrm{H}}, \infty\right]$ to the corresponding infinite regime $y \in$ $[-\infty, \infty]$

27. L. D. Landau and E. M. Liftshitz, Quantum Mechanics, 3rd ed. (Pergamon, New York, 1977), Chap. VII

28. J. Heading, An Introduction to Phase Integral Methods (Wiley, New York, 1962)

29. C. M. Bender and S. A. Orszag, Advanced Mathematical Methods for Scientists and Engineers (McGraw-Hill, New York, 1978), Chap. 10

30. The phase shift of $\frac{1}{4} \pi$ in the WKB integral relation (3) reflects the fact that the radially-dependent potential (9) of the composed black-hole-massive-field system has a classical turning point at $y_{-}=-\infty$. The WKB wave field in the classically allowed region $\left[y_{-}, y_{+}\right]$should therefore be matched only once (at the outer classical turning point $y=y_{+}$) to the corresponding WKB wave field in the classically forbidden region $y>y_{+}$of the black-hole spacetime. The standard matching procedure of the wave field across the outer classical turning point $y=y_{+}$[see Eq. (20) below] yields the familiar phase shift of $\frac{1}{4} \pi$ in the second-order WKB resonance relation (13) [27-29]

31. Here we have used the strong inequality $1 / x_{+} \gg 1$ [see Eqs. (12) and (20), and also Eq. (24) below]

32. The analytically derived expression (25) for the existence-line of the field theory corresponds to the fundamental $(n=0)$ mode of the resonance spectrum (24) 